OPEN ACCESS

Edited by:

Weidong Cai,

The University of Sydney, Australia

Reviewed by:

Minghao Dong,

Xidian University, China

Dominic T. Cheng,

Auburn University, United States

*Correspondence:

Wenbin Guo

guowenbin76@csu.edu.cn

Specialty section:

This article was submitted to

Brain Imaging Methods,

a section of the journal

Frontiers in Neuroscience

Received: 27 October 2018 Accepted: 08 February 2019

Published: 26 February 2019

Citation: Ding Y, Ou Y, Su Q, Pan P, Shan X, Chen J, Liu F, Zhang Z, Zhao J and Guo W (2019) Enhanced Global-Brain Functional Connectivity in the Left Superior Frontal Gyrus as a Possible

Endophenotype for Schizophrenia.

Front. Neurosci. 13:145.

doi: 10.3389/fnins.2019.00145

\section{Enhanced Global-Brain Functional Connectivity in the Left Superior Frontal Gyrus as a Possible Endophenotype for Schizophrenia}

Yudan Ding1, Yangpan Ou', Qinji Su², Pan Pan', Xiaoxiao Shan', Jindong Chen', Feng Liu', Zhikun Zhang', Jingping Zhao ${ }^{1}$ and Wenbin Guo ${ }^{1 *}$

${ }^{1}$ Department of Psychiatry, The Second Xiangya Hospital of Central South University, Changsha, China, ${ }^{2}$ Mental Health Center, The Second Affiliated Hospital of Guangxi Medical University, Nanning, China, ${ }^{3}$ Department of Radiology, Tianjin Medical University General Hospital, Tianjin, China

The notion of dysconnectivity in schizophrenia has been put forward for many years and results in substantial attempts to explore altered functional connectivity (FC) within different networks with inconsistent results. Clinical, demographical, and methodological heterogeneity may contribute to the inconsistency. Forty-four patients with first-episode, drug-naive schizophrenia, 42 unaffected siblings of schizophrenia patients and 44 healthy controls took part in this study. Global-brain FC (GFC) was employed to analyze the imaging data. Compared with healthy controls, patients with schizophrenia and unaffected siblings shared enhanced GFC in the left superior frontal gyrus (SFG). In addition, patients had increased GFC mainly in the thalamo-cortical network, including the bilateral thalamus, bilateral posterior cingulate cortex (PCC)/precuneus, left superior medial prefrontal cortex (MPFC), right angular gyrus, and right SFG/middle frontal gyrus and decreased GFC in the left ITG/cerebellum Crus I. No other altered GFC values were observed in the siblings group relative to the control group. Further ROC analysis showed that increased GFC in the left SFG could separate the patients or the siblings from the controls with acceptable sensitivities. Our findings suggest that increased GFC in the left SFG may serve as a potential endophenotype for schizophrenia.

Keywords: schizophrenia, global-brain functional connectivity, functional magnetic resonance imaging, endophenotype, network

\section{INTRODUCTION}

Characterized by disturbances of perception (Yoon et al., 2008), cognition (Barch and Csernansky, 2007), emotion (Holt et al., 2011), and thought (Corlett et al., 2007), schizophrenia is a devastating and complex mental disorder, affecting adults as well as adolescence with highly heterogeneous and multifaceted clinical syndromes instead of a single disease entity (Yu et al., 2017). The diagnosis of schizophrenia is largely dependent on the psychiatrists' evaluation and experience based on the comprehensive history records and laboratory examinations (Chin et al., 2018). In recent decades, great efforts have been made to identify reliable and objective biomarkers, such as electrophysiological (Turetsky et al., 2008; Smith et al., 2010; Edgar et al., 2012), neuropsychological 
(Smith et al., 2010; Edgar et al., 2012; Schulze-Rauschenbach et al., 2015), and neuroimaging indices (Edgar et al., 2012; Turner et al., 2012; Moran et al., 2013).

It has been postulated that schizophrenia is a neurodevelopmental disorder with abnormal neural connectivity of discrete brain networks and genetic and environmental factors may contribute to such dysconnectivity (Maynard et al., 2001; Karlsgodt et al., 2008). To date, substantial neuroimaging studies reveal structural and functional aberrations in many brain areas in schizophrenia or high risk populations, or both of them, including the prefrontal, cingulate, temporal, cerebellar, hippocampal, and thalamic regions (Rubinov and Bullmore, 2013; Thermenos et al., 2013; Bois et al., 2015; Chung and Cannon, 2015) within various brain networks such as the default-mode network (DMN) (Bluhm et al., 2007; Zhou et al., 2007; Ongur et al., 2010), cerebellar-cerebral networks (Konarski et al., 2005; Phillips et al., 2015), and thalamo-cortical networks (Andreasen et al., 1996; Jones, 1997; Swerdlow, 2010). Unaffected siblings of patients with schizophrenia, a subgroup of high risk subjects with approximately 50\% of genetic burden (Pergola et al., 2017), have about a 10-fold increased risk to develop schizophrenia than general population (Chang et al., 2002). Unaffected siblings are free from confounding variables caused by environmental or disease-associated factors, and thus having an advantage to assess brain function with limited confounding factors. For example, disturbed resting-state FC has been observed in the first-degree relatives (Jang et al., 2011), which was predominantly altered in schizophrenia (Lynall et al., 2010; Skudlarski et al., 2010). Therefore, similar brain abnormalities shared by patients with schizophrenia and unaffected siblings can be regarded as potential endophenotypes for schizophrenia. Endophenotypes are some heritable and characteristic changes certainly present in patients but are possible to appear in unaffected relatives. They segregate with the disease within families and can be biochemical, neuroanatomical, cognitive, endocrine, or neurophysiological parameters (Gottesman and Gould, 2003; Bertolino and Blasi, 2009).

However, results from resting-state functional magnetic resonance imaging (fMRI) of abnormal intrinsic neural activity and/or functional connectivity (FC) across brain areas within those networks were inconsistent: increased FC (Zhou et al., 2007), decreased FC (Bluhm et al., 2007), or both (Ongur et al., 2010). One possible factor accounting for the mixed findings is that the majority of neuroimaging studies adopted either seed-based region-of-interest (ROI) analysis or independent component analysis (ICA), both of which are, to some extent, dependent on prior assumptions rather than employing a wholebrain examination (McKeown et al., 2003; Mannell et al., 2010; Joel et al., 2011). Therefore, it is possible to miss the most significantly altered regions which may indicate the core pathophysiology of schizophrenia.

Another reason may be that heterogeneous samples with different illness duration and medication history have biased the findings. Results from some longitudinal MRI studies in patients with chronic schizophrenia have showed accelerated gray matter loss over time and such progressive structural alterations were more remarkable at the initial stage of illness
(Yoshida et al., 2009; Chiapponi et al., 2013;Schnack et al., 2016). As for resting-state fMRI studies, researchers have revealed reduced FC within the executive control network (ECN), DMN and dorsal attention network (DAN) in medicated patients (Woodward et al., 2011), whereas no changes were found within the ECN network in first-episode, drug-naive patients with schizophrenia (Lui et al., 2009). Therefore, it is essential to recruit first-episode, drug-naive patients with schizophrenia to explore the intact connectivity of these networks.

In the present study, we aimed to explore global-brain FC (GFC) differences by comparing a group of first-episode, drug-naive patients with schizophrenia and unaffected siblings with healthy controls employing the voxel-wise model-free GFC method, which had been described in details in our previous study (Cui et al., 2018). Apart from the seed-based ROI method and ICA method, GFC is another method of functional connectome which consists of FC of anatomically different brain areas (Craddock et al., 2013). Unlike the ROI and ICA methods, GFC is not biased by a priori specification of brain areas like ROI and spares from controversial views on the number of components in the ICA method (Kelly et al., 2012). Thus, the GFC method was preferable in our study. Based on the dysconnectivity hypothesis of schizophrenia and aforementioned studies, we hypothesized that patients with schizophrenia would reveal abnormal GFC in brain regions pertain to certain networks especially the DMN and thalamo-cortical circuit. Another hypothesis was that disrupted GFC could serve as an endophenotype shared by patients with schizophrenia and unaffected siblings. In addition, receiver operating characteristic (ROC) curve was conducted to differentiate the patients and unaffected siblings from the controls. Finally, we also examined correlations between disrupted GFC and clinical variables such as illness duration and symptom severity assessed by Positive and Negative Syndrome Scale (PANSS).

\section{MATERIALS AND METHODS}

\section{Participants}

Forty-six patients with first-episode, drug-naive patients with schizophrenia, 46 non-affected siblings of patients with schizophrenia and 46 healthy controls took part in this study. All subjects were right handed, and aged from 18 to 37 years with more than 6 years of formal education. Handedness was determined by the Annett Hand Preference Questionnaire (Dragovic and Hammond, 2007). The study was in accordance with the Helsinki Declaration and approved by the local ethics committees of the Second Affiliated Hospital of Guangxi Medical University. All participants signed their written informed consent.

The included patients and siblings were recruited from the Mental Health Center, the Second Affiliated Hospital of Guangxi Medical University in China, and the controls were recruited from the local community. The diagnosis of schizophrenia was made by two research psychiatrists (W.G. and Z.Z.) according to the Structured Clinical Interview of the 
Diagnostic and Statistical Manual of Mental Disorders-IV (DSMIV) criteria, patient edition, whereas non-patient version was used for unaffected siblings and healthy controls to rule out any psychiatric conditions. No antipsychotic medications or other psychotropic agents were treated to the patients, and PANSS total scores referring symptom severity of them was more than 70 at baseline. All participants did a series of routine physical examinations including systems review and laboratory tests to exclude any significant medical conditions and shared the same exclusion criteria: neurological disorders or history of brain injury, history of nicotine dependence, alcohol or other substance dependence, or any contraindications to MRI scan. In addition, potential controls who had a first-degree relative diagnosed with psychiatric disorders were also excluded.

\section{Imaging Acquisition and Preprocessing}

Scanning was performed on a Siemens 3.0 T scanner. Participants with soft earplugs and foam, which could reduce scanner noise and head movement, were informed to lay still and remain awake with their eyes closed. After scanning, all subjects were asked some questions to claim that they did not fall asleep during the scanning. The images were acquired with a gradientecho echo-planar imaging (EPI) sequence using the following parameters: repetition time/echo time $(\mathrm{TR} / \mathrm{TE})=2000 \mathrm{~ms} / 30 \mathrm{~ms}$, 30 slices, $64 \times 64$ matrix, $90^{\circ}$ flip angle, $24 \mathrm{~cm}$ field of view, $4 \mathrm{~mm}$ slice thickness, $0.4 \mathrm{~mm}$ slice gap, and 250 volumes lasting for $500 \mathrm{~s}$.

Software DPABI was used to preprocess the imaging data (Yan et al., 2016). After slice timing and head motion correction, participants with over $2 \mathrm{~mm}$ maximal translation and $2^{\circ}$ maximal rotation were excluded. Several covariates, including Friston-24 head motion parameters acquired through rigid body correction (de Kwaasteniet et al., 2013), signal from a ventricular region of interest, and signal from a region centered in the white matter, were removed. In addition, we applied mean frame-wise displacement (FD) according to a formula described previously (Liu et al., 2008; Power et al., 2012) to address the residual effects of motion as a covariate in group analyses. The global signal was not removed since it is still a controversial practice in the restingstate fMRI field (Hahamy et al., 2014). Then, we normalized the data to conventional EPI template in the Montreal Neurological Institute $(\mathrm{MNI})$ space at a $3 \mathrm{~mm} \times 3 \mathrm{~mm} \times 3 \mathrm{~mm}$ resolution. Finally, the images were bandpass-filtered $(0.01-0.08 \mathrm{~Hz})$ and linearly detrended following spatially smoothed with a $4 \mathrm{~mm}$ full-width at half-maximum Gaussian kernel.

\section{GFC Analysis}

Voxel-wise GFC method, defined as FC between a selected voxel and all other voxels in a given gray matter mask, was used to create voxel-to-voxel maps by composing GFC values of all voxels for each subject. SPM8 in Matlab (Liu et al., 2015) was used to generate the gray matter mask by setting the threshold at probability $>0.2$. According to Yan and colleagues (Chao-Gan and Yu-Feng, 2010), a threshold of 0.2 was used to create a gray matter mask in this study, which indicated that voxels with the probability $>0.2$ would be classified as gray matter. The GFC was computed as:

$$
G F C_{\mathrm{a}}=\Sigma_{\mathrm{b}=1}^{\mathrm{n}} \frac{r\left(T_{\mathrm{a}}, T_{\mathrm{b}}\right)}{n-1}
$$

Where, Pearson's correlation coefficient ( $r$ ) was calculated at the given voxels $\mathrm{a}$ and $\mathrm{b}$ for $T_{\mathrm{s}}$, a pair of time series, followed by Fisher r-to-z transformation (Cui et al., 2018) and the GFC of a voxel was the coefficient of this voxel with all other voxels in the mask.

\section{Statistical Analysis}

When appropriate, demographical data including age, sex, and years of education and clinical data were compared by using Chisquare test and analysis of variance (ANOVA).

After performing analysis of covariance (ANCOVA), post hoc $t$-tests were carried out to compare group differences among patients with schizophrenia, unaffected siblings, and controls. Age and the mean FD were applied as covariates in the ANCOVA and post hoc $t$-tests. The results were corrected by the Gaussian random field (GRF) theory at $p<0.05$ (voxel significance: $p<0.001$, cluster significance: $p<0.05$ ).

After identifying brain regions with abnormal GFC values showing significant differences by group comparisons, the mean GFC values were extracted from these regions for further ROC curves analysis, which was used to examine whether these regions could discriminate patients with schizophrenia or unaffected siblings from healthy controls as reliable markers.

Linear correlation analyses were performed between abnormal GFC and clinical variables in PANSS scores and illness duration in the patient group $(p<0.05)$. The Bonferroni correction was used to limit type I error.

\section{RESULTS}

\section{Demographical and Clinical Characteristics}

Two patients, 4 siblings, and 2 healthy controls were excluded due to excessive head motion. Therefore, the final analysis enrolled 44 patients, 42 non-affected siblings, and 44 healthy controls. The three groups had no significant differences in age, sex, education level, and FD values (see Table 1). The mean illness duration of the patients was $22.34 \pm 7.01$ months, and the mean PANSS total score was $90.70 \pm 11.17$.

\section{Group Differences in the GFC Values}

Compared with healthy controls, patients with schizophrenia and unaffected siblings shared enhanced GFC in the left superior frontal gyrus (SFG). In addition, as showed in Table 2 and Figure 1, the patient group had increased GFC in other areas such as the bilateral PCC/precuneus, and decreased GFC in the left ITG/cerebellum Crus I relative to the control group. No other altered GFC values were observed in the siblings group relative to the control group (Table 2 and Figure 2).

\section{Correlation Results}

After the Bonferroni correction $(p>0.05 / 7=0.007$ for abnormal GFC values in the seven brain regions), no significant 
correlations were found between GFC values and clinical variables in the patients.

\section{ROC Results}

Since the left SFG exhibited increased GFC in both the patients and the siblings, it might be considered as a marker to separate the patients or the siblings from the controls. To examine this potential, ROC analysis was conducted. As shown in Figure 3, to discriminate the patients or the siblings from the controls, the areas under the curve of the left SFG were 0.829 or 0.748 , respectively. Further diagnostic analysis showed that the sensitivity and specificity to separate the patients or siblings from the controls were 70.45 or $85.71 \%$, and 90.91 or $56.82 \%$, respectively.

\section{DISCUSSION}

In the present study, we first tested abnormalities of voxelwise brain-wide FC in first-episode, drug-naive patients with schizophrenia and non-affected siblings using the GFC analysis. The key finding was that the patients and the siblings shared enhanced GFC in the left SFG relative to the controls. Further ROC analysis showed that the GFC value in this area might serve as a marker with a relatively high sensitivity to discriminate the patients or the siblings from the controls. Compared to healthy controls, patients with schizophrenia showed disturbed GFC mainly in the thalamo-cortical network.

There are two important features of our study. First, we explored FC abnormalities in patients with schizophrenia in an unbiased way using the voxel-wise brain-wide method. To date, not a unanimous pattern of brain functional anomalies

TABLE 1 | Baseline demographic and clinical characteristics of the study participants.

\begin{tabular}{|c|c|c|c|c|}
\hline & $\begin{array}{l}\text { Patients } \\
(n=44)\end{array}$ & $\begin{array}{l}\text { Siblings } \\
(n=42)\end{array}$ & $\begin{array}{l}\text { Controls } \\
(n=44)\end{array}$ & $p$-value \\
\hline $\begin{array}{l}\text { Gender (male/ } \\
\text { female) }\end{array}$ & $28 / 16$ & $28 / 14$ & $23 / 21$ & 0.35 \\
\hline Age (years) & $23.45 \pm 4.24$ & $23.57 \pm 3.62$ & $23.55 \pm 2.58$ & 0.99 \\
\hline $\begin{array}{l}\text { Education } \\
\text { (years) }\end{array}$ & $11.11 \pm 2.46$ & $12.13 \pm 2.24$ & $11.30 \pm 1.67$ & 0.11 \\
\hline $\mathrm{FD}(\mathrm{mm})$ & $0.03 \pm 0.03$ & $0.03 \pm 0.01$ & $0.03 \pm 0.02$ & 0.34 \\
\hline $\begin{array}{l}\text { Illness duration } \\
\text { (months) }\end{array}$ & $22.34 \pm 7.01$ & & & \\
\hline
\end{tabular}

\section{PANSS}

$\begin{array}{ll}\text { Positive } & 22.48 \pm 5.37 \\ \text { symptom score } & \\ \text { Negative } & 22.50 \pm 6.38 \\ \text { symptom score } & \\ \text { General } & 45.73 \pm 6.97 \\ \text { symptom score } & \\ \text { Total score } & 90.70 \pm 11.17\end{array}$

FD, framewise displacement; PANSS, the Positive and Negative Syndrome Scale; Values are expressed as mean $\pm S D$. pertaining to schizophrenia has converged among researchers, though these studies have indicated importance of abnormalities in certain brain circuits. The reason may be that many previous studies in this field focused on some predefined brain areas using approaches based on ROI (Guo et al., 2015a). It is conceivable that different studies obtained different results by selecting different ROIs. Additionally, it is possible that the most important brain regions relating to the core pathological changes in schizophrenia were never covered in some studies. On the contrary, the GFC method used in our study investigated the FC abnormalities in a voxel-wise brain-wide and more importantly, an unbiased way.

The second important feature is the sample groups recruited in this study. First-episode, drug-naive patients with schizophrenia were recruited to explore the intact connectivity of these networks in the present study. Except patients with schizophrenia, unaffected siblings were also enrolled in the study. Taking into account that schizophrenia is a highly heritable and complex disorder, unaffected siblings of schizophrenia patients who share remarkable genetic backgrounds with the patients are at a high-risk state to develop the disease (Jang et al., 2011). In order to have a more comprehensive insight into the neural underpinnings of schizophrenia, it is essential to investigate this group of people without interference of clinical and treatment matters.

TABLE 2 | Baseline group comparison in levels of GFC across groups.

\begin{tabular}{lccc}
\hline Cluster & & Peak (MNI) & $\begin{array}{c}\text { Number of } \\
\text { voxels }\end{array}$ \\
\cline { 2 - 3 } & & & \\
& $\mathrm{x}$ & $\mathrm{y}$ & $\mathrm{z}$-value
\end{tabular}

Patients vs.

Controls

ITG/cerebellum

$-45$

$-42$

$-24$

55

$-4.6571$

Crus I

Bilateral

thalamus

Right angula

gyrus

Bilateral

PCC/precuneus

Left superior

MPFC

Right superior

frontal

gyrus/middle

frontal gyrus

Left superior

frontal gyrus

6

$-12$

15

50

4.2670

51

$-57$

33

139

4.6931

3

$-54$

33

67

4.0364

$-9$

54

45

66

4.4945

39

24

48

150

5.0740

Siblings vs.

Controls

Left superior

$-15$

66

9

28

4.1515 


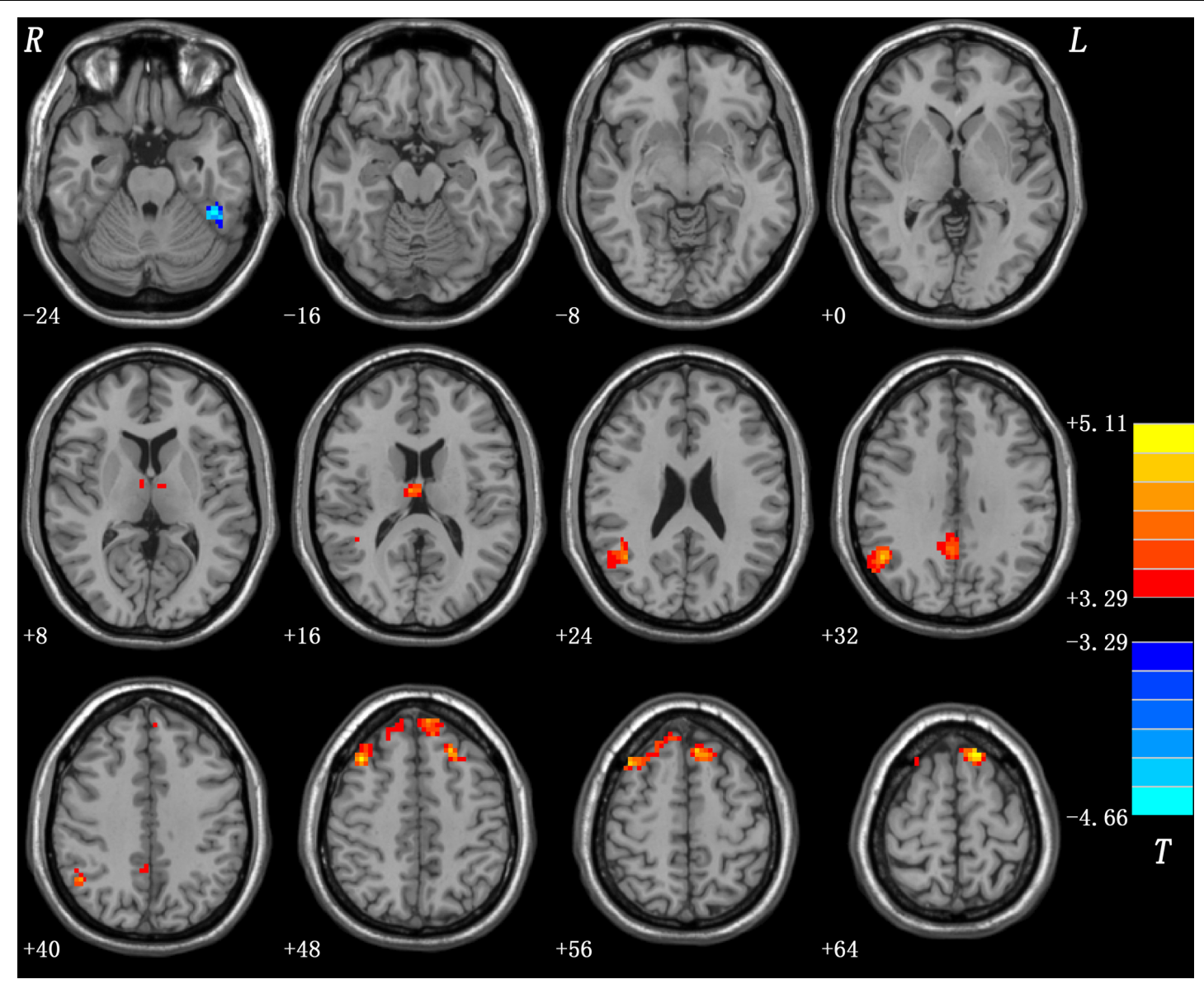

FIGURE 1 | Abnormal GFC in patients with schizophrenia relative to healthy controls. GFC, global-brain functional connectivity.

In addition, with more efforts putting into the effective treatment that could improve the clinical outcomes of patients with schizophrenia considerably, earlier identification and intervention are pushed to an urgent place (Chang et al., 2016). Investigating the vulnerability state and initial period of schizophrenia are help to address this issue.

The left SFG, involving in the impaired attention and cognitive domains (Wolf et al., 2008) including perception, working memory (Jenkins et al., 2018), and active imagery (Qiu et al., 2018), is one of the most consistently explored regions that may be a key hub in the pathophysiological processes of schizophrenia. In present study, increased GFC of the left SFG was found both in patients with schizophrenia and unaffected siblings and further ROC analysis exhibited that the GFC values of this region might be applied as a potential marker to differentiate the patients as well as the siblings from the controls with relatively high sensitivity. However, no correlations were found between the GFC value in this area and symptom severity or illness duration, which was somewhat out of our expectations. We supposed that the enhanced FC might be a trait alteration for schizophrenia independently of symptom severity and illness duration. The relatively small sample size was also a confounding factor. In addition, consistent with our results, many previous resting-state fMRI studies recorded no correlation between abnormal FC and clinical variables in patients with schizophrenia (Guo et al., 2015a). Actually, some researchers have reported a similar pattern of cognitive deficits between patients with schizophrenia and the first-degree relatives, including working memory, set shifting, and prepotent response (Johnstone et al., 2002; Brewer et al., 2005; Snitz et al., 2006). Similarly, a M100 magnetoencephalography study found greater left SFG M100 activity in not only patients with schizophrenia but also unaffected relatives (Chen et al., 2018). This shared auditory encoding abnormality indicated a compensatory adjustment by overactivating dorsal auditory pathway (Chen et al., 2013) and could also be regarded as a potential endophenotype.

The thalamus, associated with many brain functions such as cognitive and attention control (Carlesimo et al., 2011; Schmitt et al., 2017), goal-directed mental operation (Doucet et al., 2018), and experience and expression of emotion (Frodl et al., 2002), is a complex structure. Several neurobiological studies have postulated that the pathophysiology of schizophrenia involves abnormal functional interactions between the cortex and thalamus, the subcortical structure (Cheng et al., 2015). Our result of increased GFC in bilateral thalamus was consistent with previous studies, which found increased connectivity 


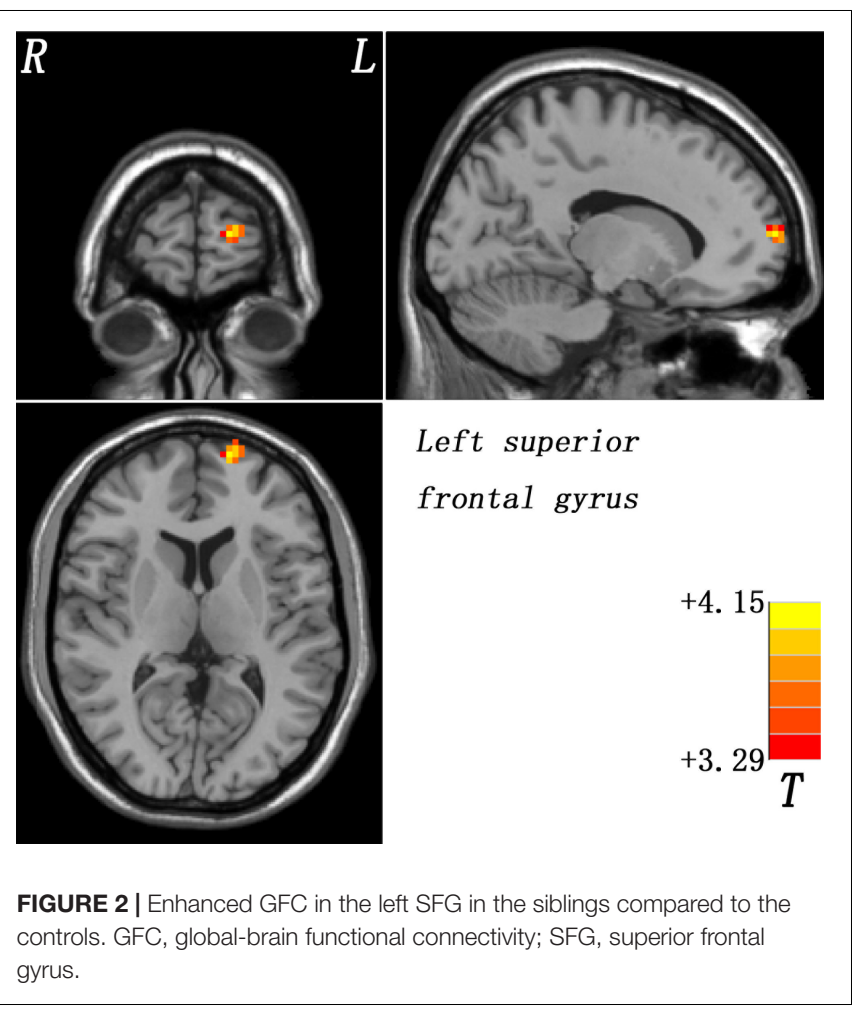

between thalamus and motor and somatosensory cortical areas (Woodward et al., 2012). Compensatory effort or dedifferentiation is always considered as an explanation of hyperconnectivity of brain regions (Cabeza et al., 2002; Grady et al., 2005; Guo et al., 2013; Su et al., 2015), which may be affected by inflammation process in the early state of schizophrenia. In that state (Anticevic et al., 2015), astrocytes could be activated by proinflammatory cytokines like interleukin6 , and consequently the metabolism and blood flow increased
(Liberto et al., 2004). It is noteworthy that numerous thalamic nuclei comprise the thalamus, and there are topographically parallel pathways linking these anatomical segregated nuclei to different cortical regions within the thalamo-cortical circuits (Alexander et al., 1986; Haber, 2003; Woodward et al., 2012). Pergola and colleagues found that gray matter volume of the mediodorsal thalamic nucleus was associated with schizophrenia but state-related, while the left anterior and midline thalamic nuclei was the most important region associated with familial risk (Pergola et al., 2017). Decreased connectivity between the prefrontal cortex and dorsomedial/anterior thalamus was also observed in previous studies (Woodward et al., 2012). It is still unclear whether the increased GFC in the bilateral thalamus documented by our study pertains to specific thalamic nuclei and whether there are associations between functional and structural imaging findings relating to thalamus. In addition, age is a vital factor that should be considered from a neurodevelopmental perspective. According to Fair and colleagues, there were significant differences in the thalamo-cortical FC between children, adolescents, and adults (Fair et al., 2010).

The DMN, including brain regions such as the posterior cingulate cortex (PCC)/precuneus, medial prefrontal cortex (MPFC), angular gyrus (Andrews-Hanna et al., 2014), and parahippocampal gyrus (Raichle et al., 2001), is one of the most consistently disturbed resting-state networks in patients with schizophrenia. MPFC is involved in the regulation of emotional behavior and self-referential processing in the DMN (Chen et al., 2012; Yu et al., 2014) and the angular gyrus plays an important role in the language process, spatial cognition, and memory retrieval (Uddin et al., 2010). Therefore, disturbed DMN network connectivity may be linked to part of poor performance seen in patients with schizophrenia. Some researchers also found unaffected siblings having altered regional activity in certain brain areas of the DMN (Guo et al., 2014a,c). However, one study showed no marked FC difference within the DMN between patients with schizophrenia and controls (Wolf et al., 2011). The

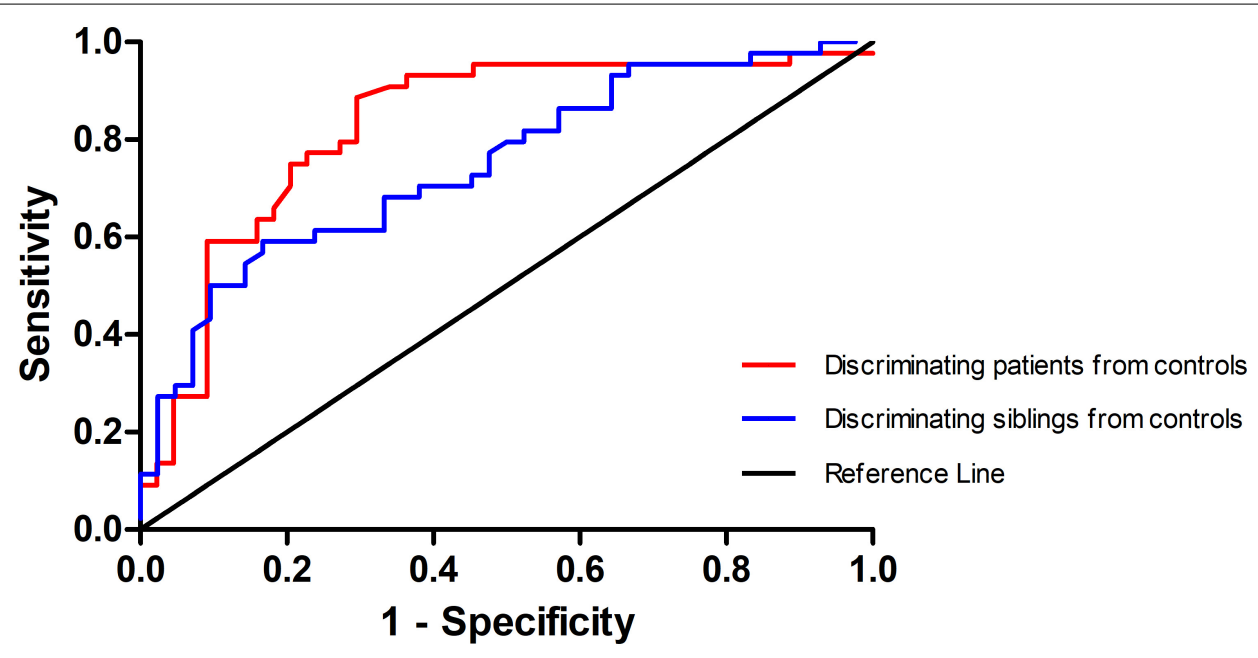

FIGURE 3 | Receiver operating characteristic (ROC) curve of separating the patients and the siblings from the controls by using the GFC values in the left SFG. GFC, global-brain functional connectivity; SFG, superior frontal gyrus. 
inconsistency may result from sample heterogeneity, sample size, and analysis methods. For patients with schizophrenia, illness duration and potential medication effects are also confounding factors. Consistent with our results, the ITG, important for emotional processing, social cognition (Guo et al., 2014b), and facial perception (Schultz et al., 2000), has been reported to have reduced FC in patients with schizophrenia as compared with healthy controls (Vercammen et al., 2010). Previous evidence also suggests that the impairment of temporal lobe and constituent parts in schizophrenia patients may be an important element in the emergence of auditory hallucinations and thought disorder (Seok et al., 2007). Intriguingly, one study suggested that deficit schizophrenia, a subgroup of patients with poorer treatment response and greater possibility to become chronicity compared to non-deficit schizophrenia, demonstrated structural and functional abnormalities in ITG (Yu et al., 2017).

In addition to the relatively small sample size, there are some limitations in this study. First, the scanning did not conduct again in the patients group after treatment. A longitudinal study is better to portray the continuous GFC alteration of brain networks in vulnerable people and patients with schizophrenia. Second, structural alterations, including gray matter and white matter, were not examined in this study. According to some researchers (Guo et al., 2012, 2015b), there were structural alterations in the gray matter and white matter in patients with schizophrenia. Hence, structural alterations underlying GFC remain unclear. However, the neuroimaging data of patients, siblings, and controls were preprocessed in the same way in order to minimize the effects caused by lack of structural examination in the present study. Finally, the study was based on resting-state fMRI without tasks involved. Therefore, it may restrict the generalizability of this study and the interpretation of underlying pathophysiology should be caution.

\section{REFERENCES}

Alexander, G. E., DeLong, M. R., and Strick, P. L. (1986). Parallel organization of functionally segregated circuits linking basal ganglia and cortex. Annu. Rev. Neurosci. 9, 357-381. doi: 10.1146/annurev.ne.09.030186.002041

Andreasen, N. C., O’Leary, D. S., Cizadlo, T., Arndt, S., Rezai, K., Ponto, L. L., et al. (1996). Schizophrenia and cognitive dysmetria: a positronemission tomography study of dysfunctional prefrontal-thalamic-cerebellar circuitry. Proc. Natl. Acad. Sci. U.S.A. 93, 9985-9990. doi: 10.1073/pnas.93. 18.9985

Andrews-Hanna, J. R., Smallwood, J., and Spreng, R. N. (2014). The default network and self-generated thought: component processes, dynamic control, and clinical relevance. Ann. N. Y. Acad. Sci. 1316, 29-52. doi: 10.1111/nyas. 12360

Anticevic, A., Hu, X., Xiao, Y., Hu, J., Li, F., Bi, F., et al. (2015). Early-course unmedicated schizophrenia patients exhibit elevated prefrontal connectivity associated with longitudinal change. J. Neurosci. 35, 267-286. doi: 10.1523/ JNEUROSCI.2310-14.2015

Barch, D. M., and Csernansky, J. G. (2007). Abnormal parietal cortex activation during working memory in schizophrenia: verbal phonological coding disturbances versus domain-general executive dysfunction. Am. J. Psychiatry 164, 1090-1098. doi: 10.1176/ajp.2007.164.7.1090

\section{CONCLUSION}

In summary, this study is the first to explore voxel-wise brainwide FC in first-episode drug-naive patients with schizophrenia and unaffected siblings. Dysconnectivity of the thalamo-cortical circuits may involve in the etiology of schizophrenia. Enhanced GFC in left SFG may serve as a potential endophenotype for schizophrenia.

\section{DATA AVAILABILITY}

All datasets generated for this study are included in the manuscript and/or the supplementary files.

\section{AUTHOR CONTRIBUTIONS}

WG and JZ designed the study. WG, QS, ZZ, YD, YO, and PP collected the original imaging data. WG, FL, XS, JC, and JZ managed and analyzed the imaging data. WG and YD wrote the first draft of the manuscript. All the authors contributed to and approved the final manuscript.

\section{FUNDING}

This study was supported by grants from the National Key R\&D Program of China (2016YFC1307100 and 2016YFC1306900) and the National Natural Science Foundation of China (Grant Nos. $81571310,81630033,81771447,81501451$, and 81471363).

\section{ACKNOWLEDGMENTS}

The authors thank all individuals who served as the research participants.

Bertolino, A., and Blasi, G. (2009). The genetics of schizophrenia. Neuroscience 164, 288-299. doi: 10.1016/j.neuroscience.2009.04.038

Bluhm, R. L., Miller, J., Lanius, R. A., Osuch, E. A., Boksman, K., Neufeld, R. W., et al. (2007). Spontaneous low-frequency fluctuations in the BOLD signal in schizophrenic patients: anomalies in the default network. Schizophr. Bull. 33, 1004-1012. doi: 10.1093/schbul/sbm052

Bois, C., Whalley, H. C., McIntosh, A. M., and Lawrie, S. M. (2015). Structural magnetic resonance imaging markers of susceptibility and transition to schizophrenia: a review of familial and clinical high risk population studies. J. Psychopharmacol. 29, 144-154. doi: 10.1177/0269881114541015

Brewer, W. J., Francey, S. M., Wood, S. J., Jackson, H. J., Pantelis, C., Phillips, L. J., et al. (2005). Memory impairments identified in people at ultra-high risk for psychosis who later develop first-episode psychosis. Am. J. Psychiatry 162, 71-78. doi: 10.1176/appi.ajp.162.1.71

Cabeza, R., Anderson, N. D., Locantore, J. K., and McIntosh, A. R. (2002). Aging gracefully: compensatory brain activity in high-performing older adults. Neuroimage 17, 1394-1402. doi: 10.1006/nimg.2002.1280

Carlesimo, G. A., Lombardi, M. G., and Caltagirone, C. (2011). Vascular thalamic amnesia: a reappraisal. Neuropsychologia 49, 777-789. doi: 10.1016/ j.neuropsychologia.2011.01.026

Chang, C. J., Chen, W. J., Liu, S. K., Cheng, J. J., Yang, W. C., Chang, H. J., et al. (2002). Morbidity risk of psychiatric disorders among the first degree 
relatives of schizophrenia patients in Taiwan. Schizophr. Bull. 28, 379-392. doi: 10.1093/oxfordjournals.schbul.a006947

Chang, M., Womer, F. Y., Bai, C., Zhou, Q., Wei, S., Jiang, X., et al. (2016). Voxel-based morphometry in individuals at genetic high risk for schizophrenia and patients with schizophrenia during their first episode of psychosis. PLoS One 11:e0163749. doi: 10.1371/journal.pone. 0163749

Chao-Gan, Y., and Yu-Feng, Z. (2010). DPARSF: a MATLAB toolbox for "Pipeline" data analysis of resting-state fMRI. Front. Syst. Neurosci. 4:13. doi: 10.3389/ fnsys. 2010.00013

Chen, J. D., Liu, F., Xun, G. L., Chen, H. F., Hu, M. R., Guo, X. F., et al. (2012). Early and late onset, first-episode, treatment-naive depression: same clinical symptoms, different regional neural activities. J. Affect. Disord. 143, 56-63. doi: $10.1016 /$ j.jad.2012.05.025

Chen, Y. H., Edgar, J. C., Huang, M., Hunter, M. A., Epstein, E., Howell, B., et al. (2013). Frontal and superior temporal auditory processing abnormalities in schizophrenia. Neuroimage Clin. 2, 695-702. doi: 10.1016/j.nicl.2013. 05.002

Chen, Y. H., Howell, B., Edgar, J. C., Huang, M., Kochunov, P., Hunter, M. A., et al. (2018). Associations and heritability of auditory encoding, gray matter, and attention in schizophrenia. Schizophr. Bull. doi: 10.1093/schbul/sby111 [Epub ahead of print].

Cheng, W., Palaniyappan, L., Li, M., Kendrick, K. M., Zhang, J., Luo, Q., et al. (2015). Voxel-based, brain-wide association study of aberrant functional connectivity in schizophrenia implicates thalamocortical circuitry. NPJ Schizophr. 1:15016. doi: 10.1038/npjschz.2015.16

Chiapponi, C., Piras, F., Fagioli, S., Piras, F., Caltagirone, C., and Spalletta, G. (2013). Age-related brain trajectories in schizophrenia: a systematic review of structural MRI studies. Psychiatry Res. 214, 83-93. doi: 10.1016/j.pscychresns. 2013.05.003

Chin, R., You, A. X., Meng, F., Zhou, J., and Sim, K. (2018). Recognition of schizophrenia with regularized support vector machine and sequential region of interest selection using structural magnetic resonance imaging. Sci. Rep. 8:13858. doi: 10.1038/s41598-018-32290-32299

Chung, Y., and Cannon, T. D. (2015). Brain imaging during the transition from psychosis prodrome to schizophrenia. J. Nerv. Ment. Dis. 203, 336-341. doi: 10.1097/NMD.0000000000000286

Corlett, P. R., Murray, G. K., Honey, G. D., Aitken, M. R., Shanks, D. R., Robbins, T. W., et al. (2007). Disrupted prediction-error signal in psychosis: evidence for an associative account of delusions. Brain 130(Pt 9), 2387-2400. doi: 10.1093/ brain/awm 173

Craddock, R. C., Jbabdi, S., Yan, C. G., Vogelstein, J. T., Castellanos, F. X., Di Martino, A., et al. (2013). Imaging human connectomes at the macroscale. Nat. Methods 10, 524-539. doi: 10.1038/nmeth.2482

Cui, X., Liu, F., Chen, J., Xie, G., Wu, R., Zhang, Z., et al. (2018). Voxel-wise brainwide functional connectivity abnormalities in first-episode, drug-naive patients with major depressive disorder. Am. J. Med. Genet B Neuropsychiatr. Genet. 177, 447-453. doi: 10.1002/ajmg.b.32633

de Kwaasteniet, B., Ruhe, E., Caan, M., Rive, M., Olabarriaga, S., Groefsema, M., et al. (2013). Relation between structural and functional connectivity in major depressive disorder. Biol. Psychiatry 74, 40-47. doi: 10.1016/j.biopsych.2012. 12.024

Doucet, G. E., Moser, D. A., Luber, M. J., Leibu, E., and Frangou, S. (2018). Baseline brain structural and functional predictors of clinical outcome in the early course of schizophrenia. Mol. Psychiatry doi: 10.1038/s41380-0180269-0

Dragovic, M., and Hammond, G. (2007). A classification of handedness using the Annett Hand Preference Questionnaire. Br. J. Psychol. 98(Pt 3), 375-387. doi: 10.1348/000712606X146197

Edgar, J. C., Hunter, M. A., Huang, M., Smith, A. K., Chen, Y., Sadek, J., et al. (2012). Temporal and frontal cortical thickness associations with M100 auditory activity and attention in healthy controls and individuals with schizophrenia. Schizophr. Res. 140, 250-257. doi: 10.1016/j.schres.2012. 06.009

Fair, D. A., Bathula, D., Mills, K. L., Dias, T. G., Blythe, M. S., Zhang, D., et al. (2010). Maturing thalamocortical functional connectivity across development. Front. Syst. Neurosci. 4:10. doi: 10.3389/fnsys.2010.00010
Frodl, T., Meisenzahl, E. M., Zetzsche, T., Born, C., Groll, C., Jager, M., et al. (2002). Hippocampal changes in patients with a first episode of major depression. Am. J. Psychiatry 159, 1112-1118. doi: 10.1176/appi.ajp.159.7.1112

Gottesman, I. I., and Gould, T. D. (2003). The endophenotype concept in psychiatry: etymology and strategic intentions. Am. J. Psychiatry 160, 636-645. doi: 10.1176/appi.ajp.160.4.636

Grady, C. L., McIntosh, A. R., and Craik, F. I. (2005). Task-related activity in prefrontal cortex and its relation to recognition memory performance in young and old adults. Neuropsychologia 43, 1466-1481. doi: 10.1016/j. neuropsychologia.2004.12.016

Guo, W., Liu, F., Chen, J., Wu, R., Zhang, Z., Yu, M., et al. (2015a). Resting-state cerebellar-cerebral networks are differently affected in first-episode, drug-naive schizophrenia patients and unaffected siblings. Sci. Rep. 5:17275. doi: 10.1038/ srep17275

Guo, W., Liu, F., Liu, J., Yu, L., Zhang, J., Zhang, Z., et al. (2015b). Abnormal causal connectivity by structural deficits in first-episode, drug-naive schizophrenia at rest. Schizophr. Bull. 41, 57-65. doi: 10.1093/schbul/sbu126

Guo, W., Liu, F., Liu, J., Yu, L., Zhang, Z., Zhang, J., et al. (2013). Is there a cerebellar compensatory effort in first-episode, treatment-naive major depressive disorder at rest? Prog. Neuropsychopharmacol. Biol. Psychiatry 46, 13-18. doi: 10.1016/j. pnpbp.2013.06.009

Guo, W., Liu, F., Liu, Z., Gao, K., Xiao, C., Chen, H., et al. (2012). Right lateralized white matter abnormalities in first-episode, drug-naive paranoid schizophrenia. Neurosci. Lett. 531, 5-9. doi: 10.1016/j.neulet.2012.09.033

Guo, W., Liu, F., Zhang, J., Zhang, Z., Yu, L., Liu, J., et al. (2014a). Abnormal default-mode network homogeneity in first-episode, drug-naive major depressive disorder. PLoS One 9:e91102. doi: 10.1371/journal.pone. 0091102

Guo, W., Su, Q., Yao, D., Jiang, J., Zhang, J., Zhang, Z., et al. (2014b). Decreased regional activity of default-mode network in unaffected siblings of schizophrenia patients at rest. Eur. Neuropsychopharmacol. 24, 545-552. doi: 10.1016/j.euroneuro.2014.01.004

Guo, W., Yao, D., Jiang, J., Su, Q., Zhang, Z., Zhang, J., et al. (2014c). Abnormal default-mode network homogeneity in first-episode, drug-naive schizophrenia at rest. Prog. Neuropsychopharmacol. Biol. Psychiatry 49, 16-20. doi: 10.1016/j. pnpbp.2013.10.021

Haber, S. N. (2003). The primate basal ganglia: parallel and integrative networks. J. Chem. Neuroanat. 26, 317-330. doi: 10.1016/j.jchemneu.2003.10.003

Hahamy, A., Calhoun, V., Pearlson, G., Harel, M., Stern, N., Attar, F., et al. (2014). Save the global: global signal connectivity as a tool for studying clinical populations with functional magnetic resonance imaging. Brain Connect. 4, 395-403. doi: 10.1089/brain.2014.0244

Holt, D. J., Lakshmanan, B., Freudenreich, O., Goff, D. C., Rauch, S. L., and Kuperberg, G. R. (2011). Dysfunction of a cortical midline network during emotional appraisals in schizophrenia. Schizophr. Bull. 37, 164-176. doi: 10. $1093 / \mathrm{schbul} / \mathrm{sbp} 067$

Jang, J. H., Jung, W. H., Choi, J. S., Choi, C. H., Kang, D. H., Shin, N. Y., et al. (2011). Reduced prefrontal functional connectivity in the default mode network is related to greater psychopathology in subjects with high genetic loading for schizophrenia. Schizophr. Res. 127, 58-65. doi: 10.1016/j.schres.2010. 12.022

Jenkins, L. M., Bodapati, A. S., Sharma, R. P., and Rosen, C. (2018). Working memory predicts presence of auditory verbal hallucinations in schizophrenia and bipolar disorder with psychosis. J. Clin. Exp. Neuropsychol. 40, 84-94. doi: 10.1080/13803395.2017.1321106

Joel, S. E., Caffo, B. S., van Zijl, P. C., and Pekar, J. J. (2011). On the relationship between seed-based and ICA-based measures of functional connectivity. Magn. Reson. Med. 66, 644-657. doi: 10.1002/mrm.22818

Johnstone, E. C., Lawrie, S. M., and Cosway, R. (2002). What does the Edinburgh high-risk study tell us about schizophrenia? Am. J. Med. Genet. 114, 906-912. doi: 10.1002/ajmg.b.10304

Jones, E. G. (1997). Cortical development and thalamic pathology in schizophrenia. Schizophr. Bull. 23, 483-501. doi: 10.1093/schbul/23.3.483

Karlsgodt, K. H., Sun, D., Jimenez, A. M., Lutkenhoff, E. S., Willhite, R., van Erp, T. G., et al. (2008). Developmental disruptions in neural connectivity in the pathophysiology of schizophrenia. Dev. Psychopathol. 20, 1297-1327. doi: 10.1017/S095457940800062X 
Kelly, C., Biswal, B. B., Craddock, R. C., Castellanos, F. X., and Milham, M. P. (2012). Characterizing variation in the functional connectome: promise and pitfalls. Trends Cogn. Sci. 16, 181-188. doi: 10.1016/j.tics.2012. 02.001

Konarski, J. Z., McIntyre, R. S., Grupp, L. A., and Kennedy, S. H. (2005). Is the cerebellum relevant in the circuitry of neuropsychiatric disorders? J. Psychiatry Neurosci. 30, 178-186.

Liberto, C. M., Albrecht, P. J., Herx, L. M., Yong, V. W., and Levison, S. W. (2004). Pro-regenerative properties of cytokine-activated astrocytes. J. Neurochem. 89, 1092-1100. doi: 10.1111/j.1471-4159.2004.02420.x

Liu, F., Zhu, C., Wang, Y., Guo, W., Li, M., Wang, W., et al. (2015). Disrupted cortical hubs in functional brain networks in social anxiety disorder. Clin. Neurophysiol. 126, 1711-1716. doi: 10.1016/j.clinph.2014.11.014

Liu, Y., Liang, M., Zhou, Y., He, Y., Hao, Y., Song, M., et al. (2008). Disrupted small-world networks in schizophrenia. Brain 131(Pt 4), 945-961. doi: 10.1093/ brain/awn018

Lui, S., Deng, W., Huang, X., Jiang, L., Ma, X., Chen, H., et al. (2009). Association of cerebral deficits with clinical symptoms in antipsychotic-naive first-episode schizophrenia: an optimized voxel-based morphometry and resting state functional connectivity study. Am. J. Psychiatry 166, 196-205. doi: 10.1176/appi. ajp.2008.08020183

Lynall, M. E., Bassett, D. S., Kerwin, R., McKenna, P. J., Kitzbichler, M., Muller, U., et al. (2010). Functional connectivity and brain networks in schizophrenia. J. Neurosci. 30, 9477-9487. doi: 10.1523/JNEUROSCI.0333-10. 2010

Mannell, M. V., Franco, A. R., Calhoun, V. D., Canive, J. M., Thoma, R. J., and Mayer, A. R. (2010). Resting state and task-induced deactivation: a methodological comparison in patients with schizophrenia and healthy controls. Hum. Brain Mapp. 31, 424-437. doi: 10.1002/hbm.20876

Maynard, T. M., Sikich, L., Lieberman, J. A., and LaMantia, A. S. (2001). Neural development, cell-cell signaling, and the "two-hit" hypothesis of schizophrenia. Schizophr. Bull. 27, 457-476. doi: 10.1093/oxfordjournals.schbul. a006887

McKeown, M. J., Hansen, L. K., and Sejnowsk, T. J. (2003). Independent component analysis of functional MRI: what is signal and what is noise? Curr. Opin. Neurobiol. 13, 620-629. doi: 10.1016/j.conb.2003.09.012

Moran, M. E., Hulshoff Pol, H., and Gogtay, N. (2013). A family affair: brain abnormalities in siblings of patients with schizophrenia. Brain 136(Pt 11), 3215-3226. doi: 10.1093/brain/awt116

Ongur, D., Lundy, M., Greenhouse, I., Shinn, A. K., Menon, V., Cohen, B. M., et al. (2010). Default mode network abnormalities in bipolar disorder and schizophrenia. Psychiatry Res. 183, 59-68. doi: 10.1016/j.pscychresns.2010.04. 008

Pergola, G., Trizio, S., Di Carlo, P., Taurisano, P., Mancini, M., Amoroso, N., et al. (2017). Grey matter volume patterns in thalamic nuclei are associated with familial risk for schizophrenia. Schizophr. Res. 180, 13-20. doi: 10.1016/j.schres. 2016.07.005

Phillips, J. R., Hewedi, D. H., Eissa, A. M., and Moustafa, A. A. (2015). The cerebellum and psychiatric disorders. Front. Public Health 3:66. doi: 10.3389/ fpubh.2015.00066

Power, J. D., Barnes, K. A., Snyder, A. Z., Schlaggar, B. L., and Petersen, S. E. (2012). Spurious but systematic correlations in functional connectivity MRI networks arise from subject motion. Neuroimage 59, 2142-2154. doi: 10.1016/j. neuroimage.2011.10.018

Qiu, L., Yan, H., Zhu, R., Yan, J., Yuan, H., Han, Y., et al. (2018). Correlations between exploratory eye movement, hallucination, and cortical gray matter volume in people with schizophrenia. BMC Psychiatry 18:226. doi: 10.1186/ s12888-018-1806-1808

Raichle, M. E., MacLeod, A. M., Snyder, A. Z., Powers, W. J., Gusnard, D. A., and Shulman, G. L. (2001). A default mode of brain function. Proc. Natl. Acad. Sci. U.S.A. 98, 676-682. doi: 10.1073/pnas.98.2.676

Rubinov, M., and Bullmore, E. (2013). Schizophrenia and abnormal brain network hubs. Dialogues Clin. Neurosci. 15, 339-349.

Schmitt, L. I., Wimmer, R. D., Nakajima, M., Happ, M., Mofakham, S., and Halassa, M. M. (2017). Thalamic amplification of cortical connectivity sustains attentional control. Nat. 545, 219-223. doi: 10.1038/nature22073

Schnack, H. G., van Haren, N. E., Nieuwenhuis, M., Hulshoff Pol, H. E., Cahn, W., and Kahn, R. S. (2016). Accelerated brain aging in schizophrenia: a longitudinal pattern recognition study. Am. J. Psychiatry 173, 607-616. doi: 10.1176/appi.ajp. 2015.15070922

Schultz, R. T., Gauthier, I., Klin, A., Fulbright, R. K., Anderson, A. W., Volkmar, F., et al. (2000). Abnormal ventral temporal cortical activity during face discrimination among individuals with autism and Asperger syndrome. Arch. Gen. Psychiatry 57, 331-340. doi: 10.1001/archpsyc.57.4.331

Schulze-Rauschenbach, S., Lennertz, L., Ruhrmann, S., Petrovsky, N., Ettinger, U., Pukrop, R., et al. (2015). Neurocognitive functioning in parents of schizophrenia patients: attentional and executive performance vary with genetic loading. Psychiatry Res. 230, 885-891. doi: 10.1016/j.psychres.2015. 11.031

Seok, J. H., Park, H. J., Chun, J. W., Lee, S. K., Cho, H. S., Kwon, J. S., et al. (2007). White matter abnormalities associated with auditory hallucinations in schizophrenia: a combined study of voxel-based analyses of diffusion tensor imaging and structural magnetic resonance imaging. Psychiatry Res. 156, $93-$ 104. doi: 10.1016/j.pscychresns.2007.02.002

Skudlarski, P., Jagannathan, K., Anderson, K., Stevens, M. C., Calhoun, V. D., Skudlarska, B. A., et al. (2010). Brain connectivity is not only lower but different in schizophrenia: a combined anatomical and functional approach. Biol. Psychiatry 68, 61-69. doi: 10.1016/j.biopsych.2010.03.035

Smith, A. K., Edgar, J. C., Huang, M., Lu, B. Y., Thoma, R. J., Hanlon, F. M., et al. (2010). Cognitive abilities and 50- and 100-msec paired-click processes in schizophrenia. Am. J. Psychiatry 167, 1264-1275. doi: 10.1176/appi.ajp.2010. 09071059

Snitz, B. E., Macdonald, A. W. III, and Carter, C. S. (2006). Cognitive deficits in unaffected first-degree relatives of schizophrenia patients: a meta-analytic review of putative endophenotypes. Schizophr. Bull. 32, 179-194. doi: 10.1093/ schbul/sbi048

Su, Q., Yao, D., Jiang, M., Liu, F., Jiang, J., Xu, C., et al. (2015). Increased functional connectivity strength of right inferior temporal gyrus in first-episode, drugnaive somatization disorder. Aust. N. Z. J. Psychiatry 49, 74-81. doi: 10.1177/ 0004867414553949

Swerdlow, N. R. (2010). Integrative circuit models and their implications for the pathophysiologies and treatments of the schizophrenias. Curr. Top. Behav. Neurosci. 4, 555-583. doi: 10.1007/7854_2010_48

Thermenos, H. W., Keshavan, M. S., Juelich, R. J., Molokotos, E., WhitfieldGabrieli, S., Brent, B. K., et al. (2013). A review of neuroimaging studies of young relatives of individuals with schizophrenia: a developmental perspective from schizotaxia to schizophrenia. Am. J. Med. Genet. B Neuropsychiatr. Genet. 162B, 604-635. doi: 10.1002/ajmg.b.32170

Turetsky, B. I., Greenwood, T. A., Olincy, A., Radant, A. D., Braff, D. L., Cadenhead, K. S., et al. (2008). Abnormal auditory N100 amplitude: a heritable endophenotype in first-degree relatives of schizophrenia probands. Biol. Psychiatry 64, 1051-1059. doi: 10.1016/j.biopsych.2008.06.018

Turner, J. A., Calhoun, V. D., Michael, A., van Erp, T. G., Ehrlich, S., Segall, J. M., et al. (2012). Heritability of multivariate gray matter measures in schizophrenia. Twin Res. Hum. Genet. 15, 324-335. doi: 10.1017/thg.2012.1

Uddin, L. Q., Supekar, K., Amin, H., Rykhlevskaia, E., Nguyen, D. A., Greicius, M. D., et al. (2010). Dissociable connectivity within human angular gyrus and intraparietal sulcus: evidence from functional and structural connectivity. Cereb. Cortex 20, 2636-2646. doi: 10.1093/cercor/bhq011

Vercammen, A., Knegtering, H., den Boer, J. A., Liemburg, E. J., and Aleman, A. (2010). Auditory hallucinations in schizophrenia are associated with reduced functional connectivity of the temporo-parietal area. Biol. Psychiatry 67, 912 918. doi: 10.1016/j.biopsych.2009.11.017

Wolf, N. D., Sambataro, F., Vasic, N., Frasch, K., Schmid, M., SchonfeldtLecuona, C., et al. (2011). Dysconnectivity of multiple resting-state networks in patients with schizophrenia who have persistent auditory verbal hallucinations. J. Psychiatry Neurosci. 36, 366-374. doi: 10.1503/jpn.110008

Wolf, R. C., Hose, A., Frasch, K., Walter, H., and Vasic, N. (2008). Volumetric abnormalities associated with cognitive deficits in patients with schizophrenia. Eur. Psychiatry 23, 541-548. doi: 10.1016/j.eurpsy.2008.02.002

Woodward, N. D., Karbasforoushan, H., and Heckers, S. (2012). Thalamocortical dysconnectivity in schizophrenia. Am. J. Psychiatry 169, 1092-1099. doi: 10. 1176/appi.ajp.2012.12010056

Woodward, N. D., Rogers, B., and Heckers, S. (2011). Functional resting-state networks are differentially affected in schizophrenia. Schizophr. Res. 130, 86-93. doi: 10.1016/j.schres.2011.03.010 
Yan, C. G., Wang, X. D., Zuo, X. N., and Zang, Y. F. (2016). DPABI: data processing and analysis for (resting-state) brain imaging. Neuroinformatics 14, 339-351. doi: 10.1007/s12021-016-9299-9294

Yoon, J. H., Tamir, D., Minzenberg, M. J., Ragland, J. D., Ursu, S., and Carter, C. S. (2008). Multivariate pattern analysis of functional magnetic resonance imaging data reveals deficits in distributed representations in schizophrenia. Biol. Psychiatry 64, 1035-1041. doi: 10.1016/j.biopsych.2008.07.025

Yoshida, T., McCarley, R. W., Nakamura, M., Lee, K., Koo, M. S., Bouix, S., et al. (2009). A prospective longitudinal volumetric MRI study of superior temporal gyrus gray matter and amygdala-hippocampal complex in chronic schizophrenia. Schizophr. Res. 113, 84-94. doi: 10.1016/j.schres.2009.05.004

Yu, M., Dai, Z., Tang, X., Wang, X., Zhang, X., Sha, W., et al. (2017) Convergence and divergence of brain network dysfunction in deficit and non-deficit schizophrenia. Schizophr. Bull. 43, 1315-1328. doi: 10.1093/schbul/ sbx014

Yu, R., Chien, Y. L., Wang, H. L., Liu, C. M., Liu, C. C., Hwang, T. J., et al. (2014). Frequency-specific alternations in the amplitude of low-frequency fluctuations in schizophrenia. Hum. Brain Mapp. 35, 627-637. doi: 10.1002/hbm. 22203

Zhou, Y., Liang, M., Tian, L., Wang, K., Hao, Y., Liu, H., et al. (2007). Functional disintegration in paranoid schizophrenia using resting-state fMRI. Schizophr. Res. 97, 194-205. doi: 10.1016/j.schres.2007.05.029

Conflict of Interest Statement: The authors declare that the research was conducted in the absence of any commercial or financial relationships that could be construed as a potential conflict of interest.

Copyright (c) 2019 Ding, Ou, Su, Pan, Shan, Chen, Liu, Zhang, Zhao and Guo. This is an open-access article distributed under the terms of the Creative Commons Attribution License (CC BY). The use, distribution or reproduction in other forums is permitted, provided the original author(s) and the copyright owner(s) are credited and that the original publication in this journal is cited, in accordance with accepted academic practice. No use, distribution or reproduction is permitted which does not comply with these terms. 